\title{
Pax6 Binds to Promoter Sequence Elements Associated with Immunological Surveillance and Energy Homeostasis in Brain of Aging Mice
}

\author{
Shashank Kumar Maurya Rajnikant Mishra \\ Department of Zoology, Biochemistry and Molecular Biology Laboratory, Institute of Science, \\ Banaras Hindu University, Varanasi, India
}

\section{Keywords}

Pax6 · Chromatin immunoprecipitation · Brain · Brain aging

\begin{abstract}
Background: Patients having mutations of Pax 6 bear phenotypes that match age-associated neurological disorders. Mutations affect most cellular functions such as cell division, growth, differentiation, and cell death in brain, eyes, pituitary, pineal, and pancreas. The progressive reduction in the level of Pax 6 during aging has also been observed. However, information about downstream targets of Pax 6 in brain is unclear. Therefore, it is presumed that age-dependent alterations of Pax6 may also affect cascades of promoter sequence recognition in brain during aging. Purpose: This study is aimed at studying the interaction of Pax6 with DNA sequence elements to explore alteration in gene targets and transcription networks of Pax6 in brain during aging. Methods: Chromatin immunoprecipitation with anti-Pax6 using tissue extracts of brain from newborn, young, adult, and old mice was done. Pulled DNA from brain was analysed by gene-specific polymerase chain reaction (PCR). Amplified PCR products were sequenced and analyzed. Results: Ageassociated alterations in binding to genetic sequence elements by Pax 6 were observed. Promoter analysis predicts genes involved in neuronal survival (Bdnf, Sparc), specificity of astrocyte ( $100 \beta, G f a p)$, cell-proliferation (Pcna), inflammation and immune response (interferon- $\gamma$, tumour necrosis factor-a), management of oxidative stress (Sod, Cat), and hy-
\end{abstract}

poxia ( $L d h)$. Conclusion: The Pax6 either directly or indirectly binds to promoter sequences of genes essential for immunological surveillance and energy metabolism in brain that alters during aging.

(c) 2017 S. Karger AG, Basel

\section{Introduction}

The brain is considered as an immune-privileged organ; however, the presence of lymphatic vessels in the meningeal compartment of the brain supports the involvement of peripheral lymphocytes in mediating brain immunity [1]. Mostly microglia in brain maintain homeostasis by secreting neurotrophic factors, but in case of infection or inflammation, they secrete pro-inflammatory cytokines (interferon $[I f n]-\gamma$, tumour necrosis factor $[T n f]-\alpha)$, ROS, or NO that induce neuronal damage, infiltration of lymphocytes, and secretion of anti-inflammatory cytokines transforming growth factor (TGF)- $\beta$ and interleukin-10 [2]. During aging, several genetic switches alter, including paired box 6 genes or protein, Pax6. The Pax6 has been considered a critical regulator because its mutations lead to cognitive impairments, cerebellar ataxia, absence of olfactory system and endocrine pancreas, anophthalmia and nasal hypoplasia [3], pineal hypoplasia [4], congenital hypopituitarism [5], nystagmus, impaired auditory processing and verbal working memory, autism, and mental retardation [6-9]. The re-

\section{KARGER}

(c) 2017 S. Karger AG, Basel 


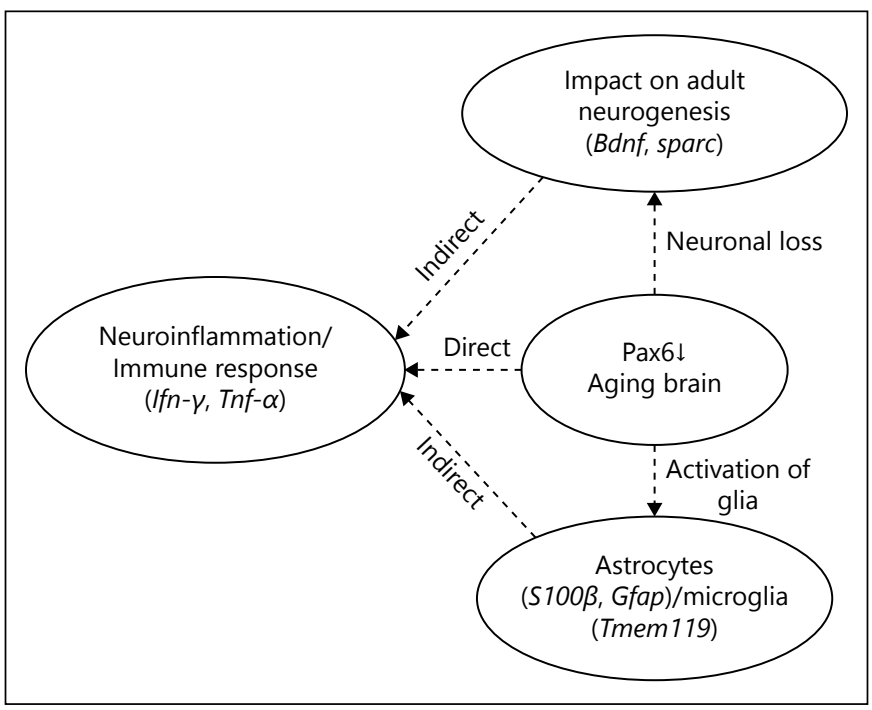

Fig. 1. Reduced level of Pax6, neural loss, glial activation, neuroinflamation or activation of glia, and neuronal loss in brain during aging may directly or indirectly affect neuroinflammation and immune response.

duced size of the corpus callosum and anterior commissure, abnormalities of the cerebral cortex and cerebellum, and absence of the pineal gland [7,10-13] have also been observed due to the mutation in Pax6. The age-associated neuronal loss, glial activation $[14,15]$, alterations in astrocytes and microglia [16], infiltration of T-cells, reduced neurogenesis [17], and Pax6-positive cells in brain [18] have also been reported. The Pax6-dependent regulation of astrocyte-such as glial development [19] and functions of Pax6 as a dual transcriptional activator and repressor [20] have also been described recently. Since the Pax6 has been found co-localised and interacting with p53, TGF- $\beta$, Smad, Sparc, and Bdnf in brain, it is presumed that Pax6 may interact with genes of microglia (Tmem119), astrocytes (S100, Gfap), pro-inflammatory cytokines (Ifn- $\gamma, \operatorname{Tnf}-\alpha)$, and anti-inflammatory cytokines $(T g f-\beta)$ to regulate cascades of genes involved in brain-specific immunity either directly or indirectly (Fig. 1). Therefore, it has been intended to explore interactions of Pax6 with sequence elements in the brain of aging mice.

\section{Methods}

Maintenance of Animals

Albino mice of AKR strain were used for the experiments. Mice were maintained at $25 \pm 2{ }^{\circ} \mathrm{C}$. They were fed upon standard mice feed in pellet form. Tap water was supplied. The life span of both male and female mice is $75 \pm 5$ weeks. Mice were maintained as per the approved guidelines of Institutional Animal Ethical Committee. The male mice were used for experiments. Their age was postnatal (P0), day 0 ; young $(\mathrm{Y}), 4 \pm 2$ weeks; adult $(\mathrm{A}), 15 \pm 4$ weeks; and old (O), $70 \pm 5$ weeks. The experiment was carried out 4 times $(n=4)$ using the brain of one mice in each age group per experiment.

\section{Chromatin Immunoprecipitation}

Chromatin immunoprecipitation (ChIP) was done using the basic method [21], with some modifications. The lysates of brain (neonate, young, adult, old) was prepared in $30 \mathrm{mM}$ Tris- $\mathrm{Cl}$ (pH 7.4), containing $1 \mathrm{mM}$ EDTA, $250 \mathrm{mM}$ sucrose, and $50 \mathrm{mM}$ mannitol. Cross linking and chromatin preparation from lysate was done by $1 \%$ formaldehyde. To stop the cross-linking reaction, $125 \mathrm{~mm}$ glycine was used. Nuclear extract was collected by centrifugation at $10,000 \mathrm{~g}$ for $10 \mathrm{~min}$. Nuclear lysis was performed in ChIP lysis buffer containing $0.1 \%$ sodium deoxycholate, $1 \%$ triton $\mathrm{x}-100,1 \mathrm{mM}$ EDTA (pH 8.0), 140 mM NaCl, 50 mM HEPES-KOH (pH 7.5) followed by sonication. The supernatant after sonication containing chromatin was incubated for $4 \mathrm{~h}$ at $4^{\circ} \mathrm{C}$ for immunoprecipitation with anti-Pax6 antibody (sc-11357, Santa-Cruz Biotech, USA). In negative control, anti-human IgG (HPO-1, Genei) was used. After centrifugation at $10,000 \mathrm{~g}$, reverse linking was performed by adding $120 \mathrm{~mm} \mathrm{NaCl}$ and incubation at $65^{\circ} \mathrm{C}$ for $1 \mathrm{~h}$. DNA obtained through immunoprecipitation was purified through phenol: chloroform purification method. The pulled DNA was checked on $1 \%$ agarose gel. The input DNA was obtained by reverse linking the chromatin prepared without antibody incubation as described above.

\section{Polymerase Chain Reaction Using Pulled DNA}

The pattern of genes in pulled DNA was analysed using genespecific primers for Pcna, S100 $\beta$, Gfap, Bdnf, Sparc, Sod, Cat, Ldh, Ifn- $\gamma$, Tnf- $\alpha$, Tmem 119 (Table 1). The polymerase chain reaction (PCR)-amplified products were evaluated on $1 \%$ agarose gel.

\section{Sequencing and Annotation of Sequencing}

Sequences were analysed using Blat (http://genome.ucsc.edu/ cgi-bin/hgBlat) and annotation using neural network promoter prediction (http://www.fruitfly.org/seq_tools/promoter.html). Predicted promoter sequences were analysed using Blastn (http:// blast.ncbi.nlm.nih.gov/Blast.cgi) and predicted genes using uniprot (http://www.uniprot.org/) and GeneCards (http://www.genecards.org/).

\section{Results}

\section{Pax6 Shows Differential Binding to Promoter}

\section{Sequence Elements in Brain}

The results suggest age-dependent alterations in binding of Pax6 with genetic sequence elements of $B d n f$, Sparc, Pcna, Ifn- $\gamma$, Tnf- $\alpha$, Sod, Cat, Ldh, Tmem119, S100 $\beta$, and Gfap (Fig. 2). The interaction of Pax6 with genetic sequence elements of Sod was found from young to old brain, whereas Cat was observed in old brain and $L d h$ in 


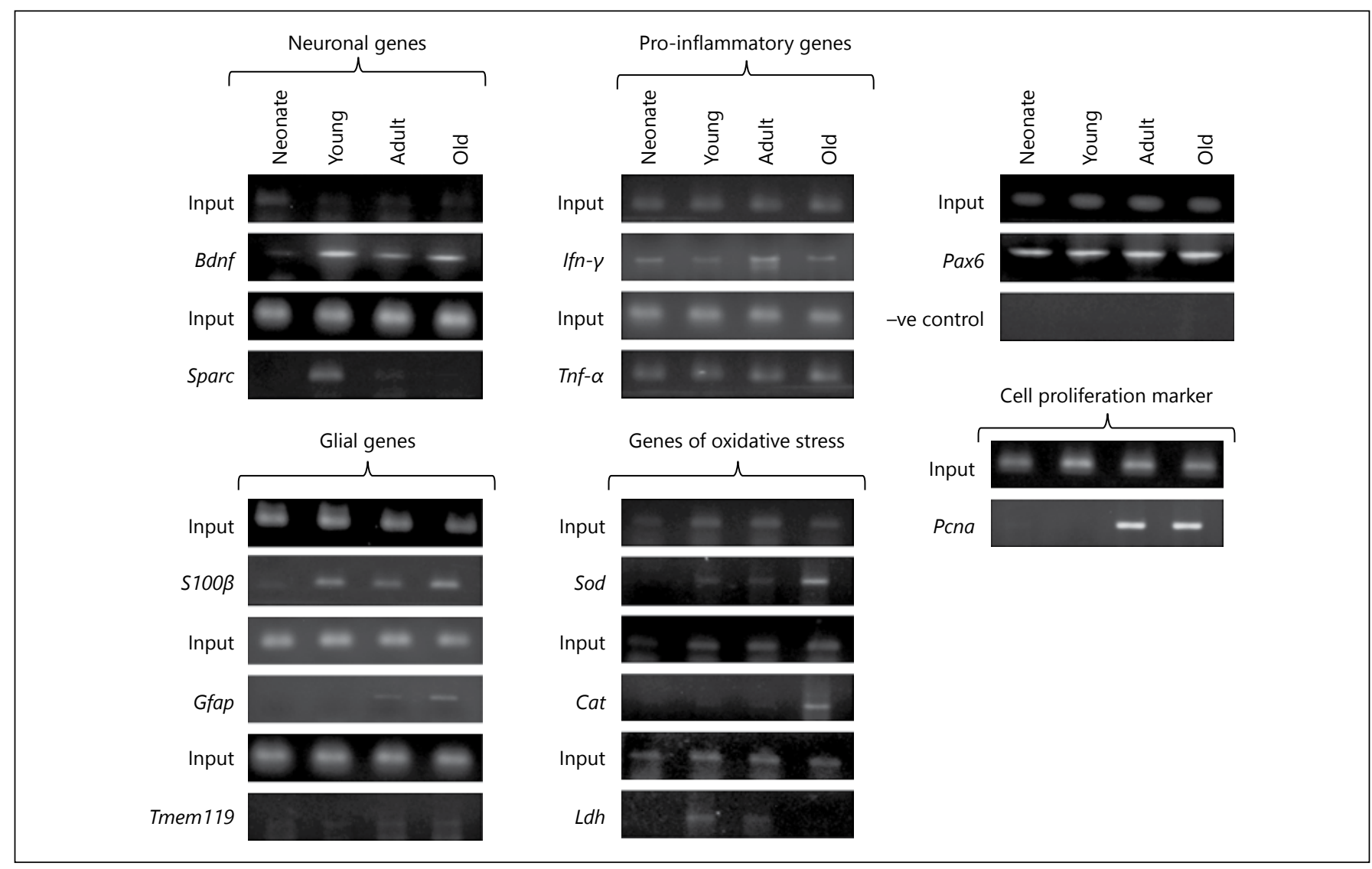

Fig. 2. It shows the status of marker genes amplified from DNA obtained through the ChIP with anti-Pax6.

Table 1. List primer pairs for identification of genes after interaction of ChIP with anti-Pax6

\begin{tabular}{llll}
\hline Forward primer & $\left(5^{\prime}-3^{\prime}\right)$ & Reverse primer & $\left(5^{\prime}-3^{\prime}\right)$ \\
\hline Pax6F & GCATGCAGAACAGTCACAGCGGAG & Pax6R & CTGTTGCTTTTCGCTAGCCAGGTT \\
Tmem $119 \mathrm{~F}$ & TCACCCAGAGCTGGTTCCATA & Tmem $119 \mathrm{R}$ & GAGGTGTCCAGGCCTTCTTC \\
$I F N-\gamma \mathrm{F}$ & AGGAAGCGGAAAAGGAGTCG & $I F N-\gamma \mathrm{R}$ & GGGTCACTGCAGCTCTGAAT \\
$S 100 \beta \mathrm{F}$ & GAGGAGCACAGCCACACTTA & $S 100 \beta \mathrm{R}$ & CATTCCCCTCCTCTGTCCT \\
GfapF & ACATCGAGATCGCCACCTAC & GfapR & TCACATCACCACGTCCTTGT \\
$P c n a \mathrm{~F}$ & GCACGTATATGCCGAGACCT & PcnaR & CAGTGGAGTGGCTTTTGGA \\
SparcF & CAAAGTGTCCACACCACCAC & SparcR & CATACGGCACAACCACACTC \\
$T N F-\alpha \mathrm{F}$ & ACGTGGAACTGGCAGAAGAG & TNF- $\alpha \mathrm{R}$ & GGGGCTCTGAGGAGTAGACA \\
$L d h \mathrm{~F}$ & CGGCTCAACCTGGT & TdhR & TAGGCACTGTCCACCAC \\
SodF & TGGGGACAATACACAAGGCTGT & SodR & TTTCCACCTTTGCCCAAGTCA \\
$C a t \mathrm{~F}$ & CCTCCTCGTTCAGGATGTGGTT & CatR & CGAGGGTCACGAA CTGTGTCAG \\
$B d n f \mathrm{~F}$ & CCGAGGTTCGGCTCACACCG & $B d n f \mathrm{R}$ & GCCCCTGCAGCCTTCCTTGG \\
\hline
\end{tabular}

young and adult brain. The interaction of Pax6 with sequences of $B d n f$ was observed in the brain of young to old age group mice, while Sparc was observed in young and adult brain, which seems to be strongly associated with neuronal health. The Pax6 was observed interacting with sequences $S 100 \beta$ from young to old age whereas Gfap was recognised only in adult and old brain. The $S 100 \beta$ is known to play a neuroprotective role in nanomolar concentration and leads to neurodegeneration when found in micromolar concentration. The GFAP being an astrocyte 
Fig. 3. It shows the promoter sequence analysis of genes amplified from DNA obtained through ChIP with anti-Pax6.

\begin{tabular}{|c|c|c|c|}
\hline \multicolumn{4}{|c|}{ Promoter predictions from sequences for Pax6: } \\
\hline Start & End & Score & Promoter sequences \\
\hline 65 & 115 & 0.35 & TGGAAGGGCACTCCCGTTTATACTGGGCTATTTTGCTTACAACTTCTGGA \\
\hline 74 & 124 & 0.27 & ACTCCCGTTTATACTGGGCTATTTTGCTTACAACTTCTGGAGTCGCCACT \\
\hline 206 & 256 & 0.23 & TGGATACCTGCAGAATTCGGGAAATGTCGCACGGCCGGGCCCCGCTGTGA \\
\hline 252 & 302 & 0.22 & GTGAGCTAGCTCTACGATCTTCTGAAGGGTGGAGTCCGGCAGTGGCCGCC \\
\hline 275 & 325 & 0.22 & GCCGGGTGGAGTCCGGCAGTGGCCGCCCGTTGACAAAGACACCACCAAGC \\
\hline 291 & 341 & 0.24 & CAGTGGCCGCCCGTTGACAAAGACACCACCAAGCTGATTCACTCCGCTGT \\
\hline \multicolumn{4}{|c|}{ Promoter predictions for Pcna: } \\
\hline Start & End & Score & Promoter sequence \\
\hline 55 & 105 & 0.36 & ACAAACAAGTAATGTGGATAAAGAAGAGGAGGCGGTAGCCATAGAGATGA \\
\hline 63 & 113 & 0.67 & GTAATGTGGATAAAGAAGAGGAGGCGGTAGCCATAGAGATGAATGAGCCT \\
\hline \multicolumn{4}{|c|}{ Promoter predictions for $5100 \beta$ : } \\
\hline Start & End & Score & Promoter sequence \\
\hline 63 & 113 & 0.18 & CAGGACCGAGAATCAAAATTCTGCTCGGCAGACTTCTCCTTTCAGGATGA \\
\hline
\end{tabular}

activation marker indicates Pax6-mediated activation of glial cells during aging. The interaction of Pax6 with sequences of Ifn- $\gamma$ and Tnf- $\alpha$ from neonate to old, and microglia-specific gene Tmem 119 was observed from young to old indicating association of Pax6 with brain-specific immunity and immunological surveillance in brain.

\section{Promoter Sequence Analysis in silico Indicates}

Genes Associated with Energy Homeostasis and

Neurodegeneration

Sequencing of PCR products of Pax6 (KT3142180), Pcna (KT 314220), and S100ß (KT 314222) and analysis indicates the presence of promoters (Fig. 3) in each sequence. Genomic BLAST suggests its presence on $5^{\prime}$-upstream and 3 '-downstream of various genes of diverse biological functions including energy homeostasis, metabolic activity, and immunity (Fig. 4). These promoters are found to be present on the $5^{\prime}$-upstream of tyrosine protein kinase Mer which transduces signals from the extracellular matrix into the cytoplasm. It has been associated with macrophage clearance of apoptotic cells, inhibition of TLRs-mediated innate immune response by activating STAT1, which selectively induces the production of suppressors of cytokine signalling SOCS1 and SOCS3. The protein kinase $\mathrm{C}$ theta type mediates non-redundant functions in TCR signalling, including $\mathrm{T}$-cell activation, proliferation, survival by mediating activation of multiple transcription factors such as NF- $\kappa B$, Jun, and NFATC1. The retinoic acid receptor and retinoid $\mathrm{X}$ receptor alpha downregulates
TGF- $\beta 1$ promoter and plays a major role in axon regeneration, during aging, cellular response to insulin, and inflammatory responses. The oxidative stress responsive serine rich protein maintains homeostasis by regulating the immune system, cellular response to hydrogen peroxide, and protein metabolism. The GATA3, transactivation enhances $\mathrm{T}$ cell receptor alpha and delta genes. It is required for Th2 differentiation process following immune and inflammatory responses. The complement component, $\mathrm{Clq}$ receptor (CD93), mediates the enhancement of phagocytosis in monocytes and macrophages. The neuronal protooncogene tyrosine protein kinase ( $\mathrm{Src}$ ) responds to ROS, TGF- $\beta$ stimulus, and regulates innate immunity. The antileukoproteinase (Slpi) modulates the innate immune response after bacterial infection. It regulates the activation of NF- $\kappa \mathrm{B}$ and inflammatory response.

These promoters are also found on Presenilin-1 (Psen1) that regulates amyloid precursor protein (APP), processing through their effects on gamma-secretase, an enzyme that cleaves APP, Annexin A5, favourable markers of $\mathrm{AD}$, restricted to glial cells, Pannexin-1, activates innate immune cells of choroid plexus and involves in cell-cell signalling, synaptic plasticity, neprilysin, abeta degrading endopeptidase clears brain amyloid protein. These promoter sequences also match to upstream of genes, which regulate metabolic activities and maintain energy homeostasis such as glutathione S-transferase omega-1, carbonic anhydrase, glucose-6-phosphate isomerase, phospholipase $\mathrm{C} \beta 3$, beta-1,3-N-acetylgalac- 
Fig. 4. Age-dependent alterations in binding of Pax6 to the promoter sequence elements of genes involved in immunological surveillance, energy homeostasis, neural development, and neurodegeneration in brain.

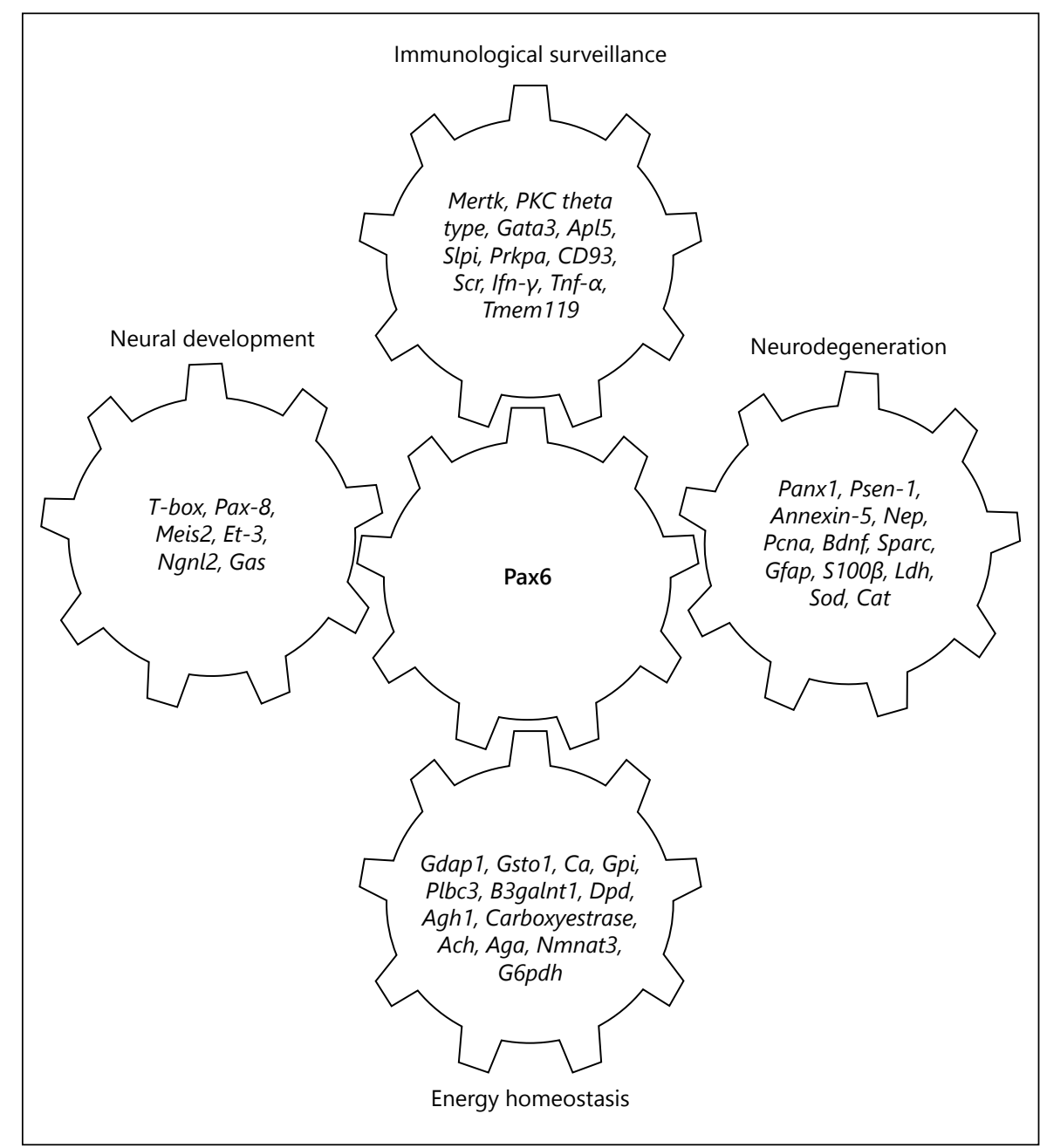

tosaminyltransferase 1 , dihydropyrimidine dehydrogenase, alcohol dehydrogenase 1, carboxylestrase, acylcarnitine hydrolase, Aspartylglucosaminidase, nicotinamide nucleotide adenylyltransferase 3, glucose-6-phosphate dehydrogenase (Fig. 4).

\section{Conclusion}

ChIP with anti-Pax6 antibody indicates age-dependent alterations in binding of Pax6 to the regulatory sequence elements of genes involved in immunological surveillance and energy homeostasis in brain during aging. Interaction of Pax6 with the regulatory element of Psen 1 also regulates the cleavage of APP and management of Alzheimer's disease. Thus, Pax6 appears as a grid to regulate cascades of genes associated with energy homeostasis, neurodegeneration, and immunological surveillance in brain.

\section{Acknowledgement}

Partial financial support from CSIR (37(1521)/12/EMR-II dated 03-04-2012) and ICMR (54/2/CFP/GER/2011-NCD-II dated 04-10-2012) are gratefully acknowledged. This article complies with the International Committee of Medical Journal editor's uniform requirements for manuscript.

\section{Authors Contribution}

R.M. planned experiments, mentored the progress of experiments, analysed results, and revised draft of the manuscript. S.K.M. worked on planned experiments, compiled and analysed data, and prepared draft of the manuscript.

\section{Disclosure Statement}

The authors declare no conflict of interest. 


\section{References}

1 Louveau A, Smirnov I, Keyes TJ, Eccles JD, Rouhani SJ, Peske JD, Derecki NC, Castle D, Mandell JW, Lee KS, Harris TH, Kipnis J: Structural and functional features of central nervous system lymphatic vessels. Nature 2015;523:337-341.

2 London A, Cohen M, Schwartz M: Microglia and monocyte-derived macrophages: functionally distinct populations that act in concert in CNS plasticity and repair. Front Cell Neurosci 2013;7:34.

3 Hill RE, Favor J, Hogan BL, Ton CC, Saunders GF, Hanson IM, Prosser J, Jordan T, Hastie $\mathrm{ND}$, van Heyningen V: Mouse small eye results from mutations in a paired-like homeobox-containing gene. Nature 1991;354:522525.

4 Hanish AE, Butman JA, Thomas F, Yao J, Han JC: Pineal hypoplasia, reduced melatonin and sleep disturbance in patients with PAX6 haploinsufficiency. J Sleep Res 2016; 25:16-22.

5 Takagi M, Nagasaki K, Fujiwara I, Ishii T, Amano N, Asakura Y: Heterozygous defects in PAX6 gene and congenital hypopituitarism. Eur J Endocrinol 2015;172:37-45.

6 Malandrini A, Mari F, Palmeri S, Gambelli S, Berti G, Bruttini M: PAX6 mutation in a family with aniridia, congenital ptosis, and mental retardation. Clin Genet 2001;60:151-154.

7 Bamiou DE, Musiek FE, Sisodiya SM, Free SL, Davies RA, Moore A, van Heyningen V, Luxon LM: Deficient auditory interhemispheric transfer in patients with PAX6 mutations. Ann Neurol 2004;56:503-509.

8 Hingorani M, Williamson KA, Moore AT, van Heyningen V: Detailed ophthalmologic evaluation of 43 individuals with PAX6 mutations. Invest Ophthalmol Vis Sci 2009;50:2581-2590.

9 Maekawa M, Iwayama Y, Nakamura K, Sato M, Toyota T, Ohnishi T, Yamada K, Miyachi T, Tsujii M, Hattori E, Maekawa N, Osumi N, Mori N, Yoshikawa T: A novel missense mutation (Leu46Val) of PAX6 found in an autistic patient. Neurosci Lett 2009;462:267-271.

10 Sisodiya SM, Free SL, Williamson KA, Mitchell TN, Willis C, Stevens JM, Kendall BE, Shorvon SD, Hanson IM, Moore AT, van Heyningen V: PAX6 haploinsufficiency causes cerebral malformation and olfactory dysfunction in humans. Nat Genet 2001;28:214-216.

11 Free SL, Mitchell TN, Williamson KA, Churchill AJ, Shorvon SD, Moore AT, van Heyningen V, Sisodiya SM: Quantitative MR image analysis in subjects with defects in the PAX6 gene. Neuroimage 2003;20:2281-2290.

12 Mitchell TN, Free SL, Williamson KA, Stevens JM, Churchill AJ, Hanson IM, Shorvon SD, Moore AT, van Heyningen V, Sisodiya SM: Polymicrogyria and absence of pineal gland due to PAX6 mutation. Ann Neurol 2003;53:658-663.

13 Ellison-Wright Z, Heyman I, Frampton I, Rubia K, Chitnis X, Ellison-Wright I, Williams SC, Suckling J, Simmons A, Bullmore E: Heterozygous PAX6 mutation, adult brain structure and fronto-striato-thalamic function in a human family. Eur J Neurosci 2004;19:1505-1512.

14 West MJ, Coleman PD, Flood DG, Troncoso JC: Differences in the pattern of hippocampal neuronal loss in normal ageing and Alzheimer's disease. Lancet 1994;344:769-772.

15 Morrison JH, Hof PR: Life and death of neurons in the aging brain. Science 1997;278: 412-429.

16 Jyothi HJ, Vidyadhara DJ, Mahadevan A Philip M, Parmar SK, Manohari SG, Shankar SK, Raju TR, Alladi PA: Aging causes morphological alterations in astrocytes and microglia in human substantia nigra pars compacta. Neurobiol Aging 2015;36:3321-3333.

17 Huang Z, Ha G, Petitto J: Reversal of neuronal atrophy: role of cellular immunity in neuroplasticity and aging. J Neurol Disord 2014; 2:pii:1000170.

18 Tripathi R, Mishra R: Aging-associated modulation in the expression of Pax6 in mouse brain. Cell Mol Neurobiol 2012;32:209-218.

19 Suzuki T, Takayama R, Sato M: Eyeless/Pax6 controls the production of glial cells in the visual center of Drosophila melanogaster. Dev Biol 2016;409:343-353.

20 Cvekl A, Callaerts P: PAX6: 25th anniversary and more to learn. Exp Eye Res 2016;pii: S0014-4835(16)30090-2.

21 Huang HS, Matevossian A, Jiang Y, Akbarian S: Chromatin immunoprecipitation in postmortem brain. J Neurosci Methods 2006;156: 284-292. 